\title{
Updates in emergency department laceration management
}

\author{
Karalynn Otterness, Adam J Singer \\ Department of Emergency Medicine, Stony Brook University Hospital, Stony Brook, NY, USA
}

Lacerations are a common reason for patients to seek medical attention, and are often acutely managed in the emergency department. Recent studies pertaining to closure techniques, sedation and analgesia, advances in wound care, and various other topics have been published, which may enhance our understanding of this injury and improve our management practices. This article will review pertinent studies published in the past few years relevant to laceration management. Understanding the current literature and appreciating which areas warrant further investigation will help us optimize outcomes for patients who sustain laceration injuries.

Keywords Lacerations; Wounds and injuries; Emergency medicine

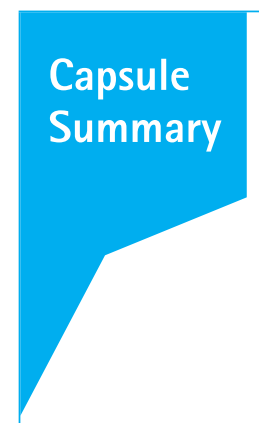

What is already known

Lacerations are a common presenting complaint for patients who seek care in emergency departments.

What is new in the current study

The field of laceration management continues to evolve, and recent studies pertaining to closure techniques, sedation and analgesia, advances in wound care, and other miscellaneous topics may enhance our understanding of this injury and improve our management practices.
elSSN: 2383-4625

Received: 3 March 2018

Revised: 18 April 2018

Accepted: 23 April 2018

Correspondence to: Karalynn Otterness Department of Emergency Medicine, Stony Brook University Hospital, HSC, Level 4, Rm 080, Stony Brook, NY 11797-8350, USA

E-mail: Karalynn.0tterness@ stonybrookmedicine.edu ORCID

http://orcid.org/0000-0001-5473-6679

\section{INTRODUCTION}

Lacerations remain a common cause of emergency department (ED) visits in the United States. They account for approximately 8.2\% of ED visits, resulting in an estimated 7 to 9 million lacerations treated in US EDs annually. ${ }^{1}$ The goals of laceration management include achieving hemostatic closure and optimizing cosmetic outcome without increasing the risk of infection and other complications. Although laceration injuries have occurred since the beginning of mankind, the field of wound management continues to evolve. This review article will focus on updates in laceration management over the past few years specifically, since 2014. It will not serve as a comprehensive review of laceration management, though several thorough reviews exist. ${ }^{2-4}$ In general, the included updates can be subdivided into various categories including wound closure techniques, sedation and analgesia, post-closure wound care, and other miscellaneous topics. 


\section{WOUND CLOSURE TECHNIQUES}

Recent studies have evaluated several aspects of wound closure including type of closure material, repair using sterile versus nonsterile gloves, irrigation technique, and suturing technique.

\section{Closure material}

A wide variety of wound closure materials exist, and choice of material depends on several factors including location of the wound, amount of tension on the wound edges, and various provider and patient factors. Many nailbed lacerations presenting to the ED are repaired using 6.0 simple interrupted absorbable sutures. However, recent data suggests that tissue adhesive glue may be equally efficacious. One systematic review of 6 articles which compared sutures to cyanoacrylate tissue adhesives for pediatric and adult nailbed injuries found that adhesives were as effective as sutures with significantly shorter time to repair. ${ }^{5}$ Another recent article on type of closure material compared cosmetic outcomes of extremity and trunk lacerations repaired with absorbable versus non-absorbable sutures. This was a randomized controlled trial of 115 pediatric and adult patients, which found similar cosmetic outcomes and no statistically significant difference in complication rates between the 2 groups, suggesting that repair of simple lacerations on the trunk and extremities with absorbable sutures could be considered a viable alternative to non-absorbable sutures. ${ }^{6}$ Although promising, further research is necessary and it remains common practice to repair these types of lacerations using non-absorbable sutures.

Two recent studies pertaining to type of closure material are from the surgical literature, but may be pertinent to ED laceration management. A randomized controlled trial of over 1,200 adults undergoing closure of laparotomy incisions assessed surgical site infection rates of traditional monofilament sutures versus monofilament sutures coated with an antimicrobial agent called triclosan. This study found no difference in infection rates. ${ }^{7}$ It is unclear whether use of antimicrobial-coated sutures would reduce infection rates after repair of ED lacerations, many of which are contaminated. Another randomized controlled trial of forty patients undergoing skin closure after implantation of cardiac devices compared traditional skin closure using sutures and Dermabond (Ethicon, Cincinnati, OH, USA) to a zip-type tape-based adjustable closure device. This device consists of 2 parallel linear adhesives joined by a zipper-like reclosable piece (Fig. 1). ${ }^{8}$ The study found that the zip-type closure group had shorter closure times with no difference in post-operative pain or scar cosmesis. ${ }^{8}$ Although further ED based studies are needed, the results seem promising and may result in shorter ED lengths of stay.

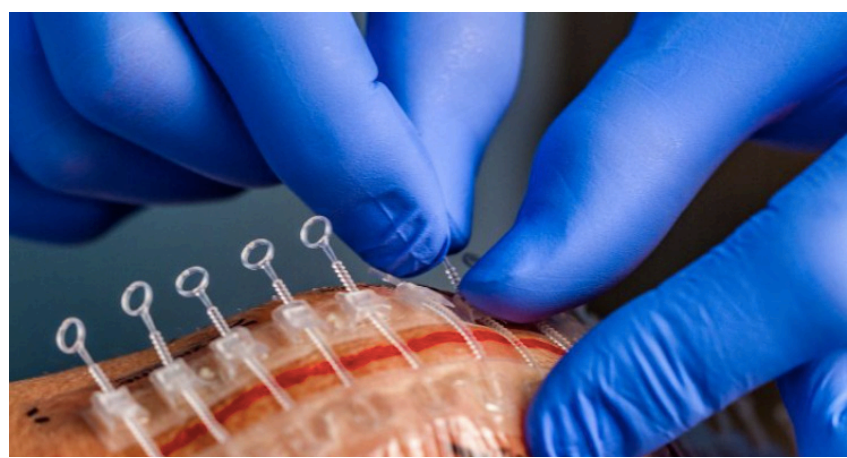

Fig. 1. Zip surgical skin closure device. Reproduced from Lalani GG, et al. Pacing Clin Electrophysiol 2016;39:1077-82, with permission was granted from Wiley Periodicals. ${ }^{8}$

\section{Sterile versus nonsterile gloves}

Laceration repair is often performed with sterile gloves, although strong evidence for this practice is lacking. Two recent studies have assessed infection rates after suture repair using sterile versus non-sterile gloves. The first is a randomized controlled trial of 493 patients undergoing minor skin excision surgeries, which found that nonsterile gloves were non-inferior to sterile gloves with regards to wound infections. ${ }^{9}$ A recent meta-analysis including 13 randomized controlled trials totaling over 11,000 patients found no difference between surgical site infection rates after outpatient cutaneous and dental procedures using sterile versus nonsterile gloves. ${ }^{10}$ While surgically created incisions are likely less contaminated than many lacerations treated in the ED, these results are similar to the findings of a large multicenter randomized controlled trial of lacerations repaired in the ED. ${ }^{11}$ Given the similar rates of infection, it is a reasonable practice to use clean, non-sterile gloves for laceration repairs in the ED.

\section{Irrigation}

Other recent studies have looked at irrigation techniques prior to wound closure. Irrigation is thought to cleanse the wound of dirt and debris and lessen the bacterial load prior to closure, though the ideal pressure and type of irrigation fluid remain controversial. A randomized controlled trial of 2,551 patients with open fractures compared high ( $>20$ psi) versus low (5-10 psi) versus very low (1-2 psi) pressure of irrigation, and also assessed irrigation with castile soap versus normal saline. Reoperation rates for infections or poor healing were similar for all pressures, and less in the normal saline group compared to the soap group. ${ }^{12}$ Another study which evaluated type of irrigation fluid included 444 ED patients with lacerations randomized to normal saline irrigation alone versus normal saline followed by povidone-iodine skin scrub. Rates of infection were lower in the normal saline only group 
(5.8\% vs. 8.7\%). ${ }^{13}$ Normal saline appears to be superior to castile soap and povidone-iodine solution as a laceration irrigation fluid.

\section{Suturing technique}

While many lacerations are repaired in the ED using simple interrupted sutures, 2 recent studies have evaluated alternative techniques. One was a Cochrane review which compared simple interrupted sutures to continuous subcuticular sutures in non-obstetric surgery. It included 5 randomized controlled trials totaling over 800 patients, and though the overall quality of the evidence was poor, the authors concluded that superficial wound dehiscence may be reduced using continuous subcuticular sutures. ${ }^{14}$ Further ED based studies are needed prior to recommending change of practice. The next study evaluated a technique called W-plasty to close facial lacerations presenting to the ED. Traditionally used for scar revision, W-plasty involves making continuous zig-zag, $W$-shaped incisions on either side of the laceration margins which results in excision of the original laceration site (Fig. 2). ${ }^{15}$ Used as a primary closure technique, the goal of $\mathrm{W}$-plasty is to minimize scarring for lacerations which deviate more than 30 degrees from the relaxed skin tension line, as this degree of deviation makes lacerations more prone to noticeable scar formation. W-plasty achieves this by dividing the laceration into several split cuts, thus changing the direction of the incision. This retrospective observational ED based study compared fifteen patients with facial lacerations repaired via simple debridement and standard suturing technique to twenty patients who underwent W-plasty technique. Patients in the W-plasty group had improved scores on the Stony Brook scar evaluation scale at both short term and long-term follow-up, without increased time to procedure completion. ${ }^{15}$ As this study was small due to loss to follow-up and all procedures were performed by a single emergency medicine provider, further studies are warranted prior to adopting this technique. While studies conducted on elective surgical incisions in the operating room may not translate to the ED repair of traumatic lacerations, a recent study is of interest. A randomized clinical trial of 560 patients with midline laparotomy incisions found that patients sutured with small ( $5 \mathrm{~mm}$ every $5 \mathrm{~mm}$ ) bites had lower dehiscence rates and similar infection rates as those closed with larger bites $(1 \mathrm{~cm} \text { every } 1 \mathrm{~cm})^{16}$

\section{SEDATION AND ANALGESIA}

\section{Sedation}

Sedation for laceration repair is most commonly done for pediatric patients, who may not otherwise cooperate for the procedure. One retrospective chart review of 237 pediatric patients with facial lacerations found that general anesthesia significantly delayed time to wound closure (880 vs. 260 minutes) and hospital length of stay compared to procedural sedation. ${ }^{17}$ Often, procedural sedation medications are administered via intramuscular or intravenous routes, which can be challenging in the pediatric patient population. Some studies have looked at alternate routes of medication administration, including oral and intranasal. A single center randomized controlled trial of 68 children with lacerations compared oral midazolam $(0.7 \mathrm{mg} / \mathrm{kg})$ to oral ketamine $(5 \mathrm{mg} / \mathrm{kg})$ and found that parental assessment of pain using the visual analog scale was similar in the 2 groups, but the sedation failure rate was higher with ketamine (32\% vs. 6\%). ${ }^{18}$ Another randomized controlled trial of 40 children with lacerations found lower pa-
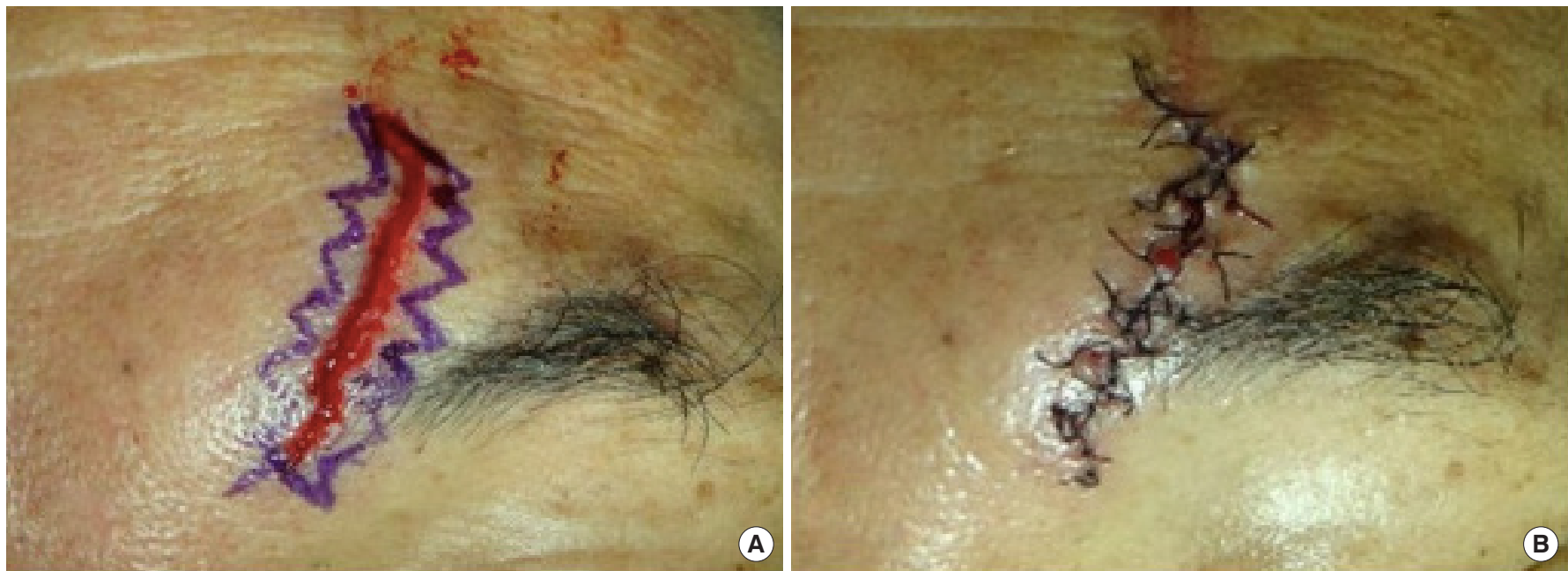

Fig. 2. W-plasty laceration repair technique. Reproduced from Min JH, et al. Am J Emerg Med 2017;35:1804-9, with permission was granted from Elsevier. $^{15}$ 
rental anxiety with intranasal dexmedetomidine compared to intranasal midazolam. ${ }^{19}$ Optimal medication choice and route of sedative remains controversial and may vary based on the individual patient and clinical situation.

\section{Analgesia}

Wound infiltration with local anesthesia is common prior to laceration repair, however this often produces pain or discomfort during subcutaneous injection. Recent studies have evaluated various adjunctive therapies to reduce this pain. While topical anesthetics are frequently used in pediatric patients requiring painful procedures, their use in adults is not as prevalent. However, recent research suggests that topical anesthetics are effective both in isolation and as an adjunct to infiltrated agents. A retrospective chart review of 89 patients with a mean age of 33 years old found that only $24 \%$ of patients receiving topical lidocaine-epinephrine-tetracaine (LET) required additional anesthesia, and these were mostly patients with longer lacerations or with lacerations in more painful locations such as extremities, trunk, fingers, and toes. ${ }^{20}$ Another study of adults with facial lacerations found addition of topical eutectic mixture of local anesthetics to routine care decreased pain and improved patient satisfaction scores. ${ }^{21}$ A Cochrane review of 25 randomized controlled trials totaling 3,278 patients compared topical versus infiltrated anesthetics and found that topical anesthetics had no complications and provided effective pain control, with some individual studies suggesting similar efficacy to local anesthesia. ${ }^{22}$ Topical anesthetics offer many advantages and may be underutilized. One study evaluated the efficacy of an educational session and triage informational poster aimed to encourage the use of LET and found increased usage and decreased time to LET after the interventions. ${ }^{23}$ The use of topical anesthetics should be strongly considered as an adjunct to other types of analgesia.

One of the oldest known anesthetic techniques is cryotherapy with ice. Recent studies have looked at ice as an adjunct to decrease pain of local anesthetic infiltration. A single center randomized controlled trial of 90 patients randomized to receive an ice pack prior to injection versus no ice pack found lower pain scores both before and after the procedure in the group receiving ice. $^{24}$ These findings were corroborated by another recent randomized controlled trial of 50 patients. ${ }^{25}$ The application of ice should be considered prior to local infiltration in order to decrease procedure-induced pain.

If local anesthetic is to be utilized, certain infiltration techniques may minimize the pain of the injection. Previous studies have evaluated mixing the anesthetic with sodium bicarbonate, and warming the anesthetic to body temperature. ${ }^{26-30}$ Although ef- fective, these techniques can be time consuming and pose additional limitations such as reducing the shelf life of the anesthetic. One recent study evaluated volunteers who received lidocaine injections with 3 different sized needles, and found that the thinnest needle (27 gauge) was the least painful. ${ }^{31}$ Another study assessed whether the speed of infiltration affected the amount of pain associated with injection, but found no difference whether the same volume was injected over 15, 30, or 45 seconds. ${ }^{32}$

Often, analgesia for repair of finger lacerations is administered via a digital nerve block in order to avoid distortion of the wound site from the edema associated with local infiltration. A commonly used approach involves injecting local anesthetic into the web space on either side of the finger, which provides analgesia to the entire digit. As the injections can be quite painful, different approaches have been investigated. One randomized controlled trial compared the efficacy of a single subcutaneous palmar injection (introduced vertically through the distal joint line of the metacarpophalangeal joint) versus the traditional 2 injection technique and found similar results in terms of pain control and time of anesthesia onset. ${ }^{33}$ An observational case control study found that patients with a history of opioid abuse who underwent digital block required a higher total amount of local anesthetic, and the time to anesthesia onset was longer. ${ }^{34}$ In light of the current opioid epidemic, it is important to recognize that many of our patients who are opioid tolerant may require more anesthetic and take longer to achieve adequate anesthesia when receiving digital nerve blocks.

\section{POST-CLOSURE WOUND CARE}

An important component of laceration management is postclosure wound care. Several recent studies have evaluated controversial issues pertaining to laceration aftercare instructions including prophylactic antibiotics, various therapies to minimize scarring, as well as type of wound dressings and dressing instructions.

\section{Antibiotics}

Much controversy and practice variation exist regarding the role of prophylactic systemic antibiotics after laceration repair. Previous studies have found no role for routine use of oral antibiotics, however most excluded certain patient populations including those with wounds considered to be high risk (bites, crush wounds, grossly contaminated wounds, and wounds involving other structures such as cartilage, tendons, joints, or bones) as well as immunocompromised populations (patients with HIV/AIDS, chemotherapy recipients, organ transplant recipients, and diabetics). ${ }^{3}$ Recent studies have reached similar conclusions, and routine use of pro- 
phylactic systemic antibiotics is likely unnecessary and should be considered on a case by case basis. A prospective observational study of 135 ED patients with simple, uncomplicated hand lacerations found that $35 \%$ received oral antibiotics with an overall wound infection rate of $4.8 \%$, with more infections developing in the group who received antibiotics. ${ }^{35}$ Another study of 330 patients with traumatic wounds which were swabbed and sent for cultures found that despite the fact that 90\% received prophylactic oral antibiotics, less than 10\% of the wounds were found to be contaminated with potentially pathologic bacteria. Furthermore only $1.2 \%$ of patients actually developed wound infections, and all of these infections occurred in patients who received antibiotics. ${ }^{36} \mathrm{~A}$ randomized, double-blinded controlled multicenter feasibility pilot trial assigned patients with simple hand lacerations to receive cephalexin, clindamycin or placebo. Of the 78 enrolled patients, only one was found on follow-up to have an infection yielding an overall rate of $1 \%$, suggesting that further studies with large sample sizes will be needed. ${ }^{37}$ Rates of postrepair wound infections are low, and prophylactic antibiotics in immunocompetent patients with low risk wounds is likely unnecessary.

One type of traumatic wound which deserves special attention is that sustained from a dog bite. A prospective multicenter observational study aimed to determine which patients would benefit from prophylactic antibiotics after sustaining a dog bite. The study found that puncture wounds and wounds which were sutured closed were more prone to infection and warrant prophylactic antibiotics. ${ }^{38}$ The routine use of prophylactic antibiotics for all dog bites is likely not beneficial, but should be considered on a case-by-case basis, especially in high risk bite wounds (such as extremity wounds).

\section{Scar minimization}

A 2015 review article highlighted various novel scar therapies. ${ }^{39}$ While the article described several topical, mechanical, and intralesional modalities for preventing and treating scars, for the purpose of this article we will focus only on therapies to prevent scar formation in the acute phase. Topical onion extract has anti-inflammatory and anti-microbial properties and has potential benefit when used prophylactically to reduce scar formation, though the evidence is inconclusive. Two additional topical agents, mitomycin $C$ and imiquimod, have been evaluated with conflicting evidence as preventative treatments for keloid scar formation.

Previously studied as a therapy for burns, honey has recently been evaluated in the literature as a topical agent for wounds to promote healing, prevent infection, and minimize scarring. A 2015 Cochrane review assessed the role of honey on various types of acute and chronic wounds and found some benefit for partial thickness burns and infected post-operative wounds, though the evidence was too heterogeneous and low quality to draw any firm overall conclusions. ${ }^{40}$ Two recent prospective randomized controlled trials in the surgical literature assessed the effect of Manuka honey on surgical scar healing but found no significant difference compared to standard treatment modalities. ${ }^{41,42}$ The role of honey in wound management is inconclusive and further investigation is warranted.

\section{Dressings}

There is significant practice variation regarding the most effective way to dress repaired lacerations. Two recent studies have addressed issues pertaining to wound dressings and aftercare instructions. A multicenter randomized controlled trial evaluated the effect of the embrace Advanced Scar Therapy (Neodyne Biosciences, Newark, CA, USA) device (a silicone based elastomeric dressing which offloads tension on the incision) (Figs. 3,4$)$ on abdominoplasty scar appearance and found that at 12 months, the portion of scars treated with the embrace device had improved scar appearance. ${ }^{43}$ As this was a surgical wound study, further research is needed to see if these results can be applied to traumatic lacerations repaired in the ED.

After wound closure, patients are often advised to leave the dressing in place for 24 hours, as covering the wound is thought to act as a protective barrier against bacterial contamination. Despite this common practice, there is some limited data supporting early removal of dressings within the first 12 hours to allow for routine bathing without increasing the infection risk. ${ }^{44} \mathrm{~A}$ Cochrane review of 3 studies including 280 patients randomized to early (within 48 hours) versus late (after 48 hours) dressing removal after surgery found no difference in infection rates. ${ }^{45}$ It should be noted that this review was in patients undergoing planned surgery and the wounds were all clean or clean-contaminated, not on traumatic wounds treated in the ED.

\section{MISCELLANEOUS}

Two recent studies pertaining to specialist consultation for laceration management have been published. One survey study of 288 patients with facial lacerations repaired either by emergency physicians or plastic surgeons found that overall satisfaction did not differ between the 2 groups, however for pediatric repairs and female patients, satisfaction was higher in the plastic surgeon repair group. Overall, ED length of stay was significantly shorter (less than half the time) for those patients in the emergency physician repair group. ${ }^{46}$ A retrospective cohort study of over 1,200 pediatric patients with facial lacerations found that fewer patients with public insurance had specialty consultation for repair 


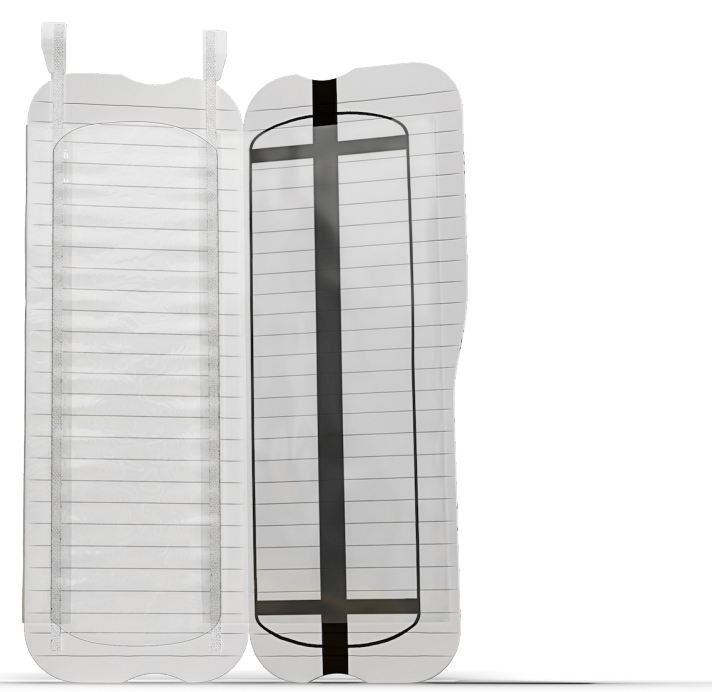

Fig. 3. Embrace Advanced Scar Therapy device. Photo courtesy of Neodyne Biosciences.

compared to those with private insurance, despite similar presentation and wound characteristics. Furthermore, those with public insurance were significantly less likely to receive procedural sedation compared to their privately insured peers. ${ }^{47}$

One randomized controlled study of 580 patients with severe dog bites to the extremity assessed the role of negative pressure wound therapy on wound healing. After debridement, patients were randomized to traditional care versus negative pressure wound therapy via a wound vacuum. The study found that negative pressure wound therapy decreased wound infection rates by more than half ( $9.1 \%$ vs. approximately $4 \%)$. Furthermore, recovery times were shorter in the negative pressure group. ${ }^{48}$ The role of negative pressure for severe bite wounds appears promising, and further research is warranted.

A retrospective chart review of 3,234 patients with facial lacerations found that this injury is more common in males than females, and that forehead followed by eyebrow and lip were the most common wound locations. A fall was the most common injury mechanism. Of those who underwent follow-up management, $3.7 \%$ had complications, most commonly wound dehiscence. ${ }^{49}$

One prospective observational study from the surgical literature recorded surgeons' conversations during wound closure for 167 patients undergoing laparotomy and classified the conversations as case relevant versus case irrelevant. The study found that case-relevant communication was associated with reduced surgical site infection, whereas case-irrelevant conversation during the procedure was associated with increased incidence of infection which suggests that distraction may contribute to post-wound closure infections. ${ }^{50}$

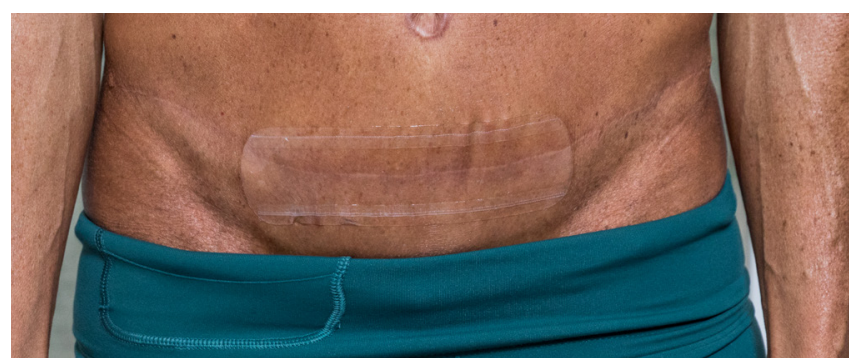

Fig. 4. Embrace Advanced Scar Therapy device applied to patient. Photo courtesy of Neodyne Biosciences.

\section{CONCLUSION}

Several studies pertaining to wound closure, sedation and analgesia, post-closure wound care, and other miscellaneous topics have been published since 2014 in the emergency medicine and surgical literature. Tissue adhesive glue is a viable option for repair of nailbed lacerations. There may be a role for absorbable sutures in repair of trunk and extremity lacerations, though further research is needed. A recent surgical study suggests that antimicrobial coated sutures do not decrease infection rates compared to traditional monofilament sutures. While thus far only used in the operating room, zip type closure devices may prove in the future to be beneficial in ED laceration repair, as they decrease time to closure without compromising scar cosmesis. Clean, non-sterile gloves do not increase wound infection rates compared to sterile gloves, and sterile saline is superior to castile soap and povidone-iodine solution as a wound irrigation fluid. Further ED based research is needed to compare standard simple interrupted suture technique to other techniques such as continuous subcuticular sutures. One study found that W-plasty improved scar cosmesis for facial lacerations repaired in the ED, though further research is warranted before adopting this technique. Sedative medication choice and administration route remain controversial, however procedural sedation results in shorter time to wound closure and total length of stay compared to general anesthesia for repair of pediatric lacerations. The use of topical anesthetics and cryotherapy with ice are beneficial in reducing pain related to laceration repair and should be more widely adopted. Smaller gauge needles are associated with decreased pain during local anesthetic infiltration, though speed of infiltration does not appear to have an effect. A single, palmar injection may be less painful than the standard 2 injection technique of digital nerve block while providing equivalent procedural anesthesia. Patients with a history of opioid abuse may require higher doses of local anesthetic and longer time to onset of anesthesia during digital nerve block. The risk of wound infection after laceration repair is low, and the rou- 
tine use of prophylactic antibiotics is unnecessary, but should be considered in high-risk patients and high-risk wounds. This is also true for prophylaxis of dog bites, although puncture wounds and bites closed in the ED appear to be at higher risk and thus antibiotics are warranted in these circumstances. The evidence for various topical agents aimed to minimize wound scarring is conflicted, and further research is necessary. A silicone-based embrace dressing improved abdominoplasty scar appearance, although the role of this type of dressing for lacerations repaired in the ED warrants further investigation. Though commonly included in aftercare instructions, little data exists for keeping a dressing in place for 24 hours after laceration repair, and limited evidence suggests that early removal has similar outcomes. Specialist consultation for laceration repair increases ED length of stay without affecting overall patient satisfaction with wound repair. Some literature suggests that publicly insured patients receive specialty consultation and procedural sedation less often than their privately insured counterparts. There may be a role for negative pressure therapy for severe dog bite wounds. Overall, lacerations appear to be more common in males, with fall being the most common mechanism and face being the most common wound location. Distractions during wound closure may contribute to a higher incidence of postoperative infection.

\section{CONFLICT OF INTEREST}

No potential conflict of interest relevant to this article was reported.

\section{REFERENCES}

1. Singer AJ, Thode HC Jr, Hollander JE. National trends in ED lacerations between 1992 and 2002. Am J Emerg Med 2006; 24:183-8.

2. Singer AJ, Dagum AB. Current management of acute cutaneous wounds. N Engl J Med 2008;359:1037-46.

3. Mankowitz SL. Laceration management. J Emerg Med 2017; 53:369-82.

4. Forsch RT, Little SH, Williams C. Laceration repair: a practical approach. Am Fam Physician 2017;95:628-36.

5. Edwards S, Parkinson L. Is fixing pediatric nail bed injuries with medical adhesives as effective as suturing?: a review of the literature. Pediatr Emerg Care 2016 Dec 12. https://doi. org/10.1097/PEC.00000000000000994.

6. Tejani $C$, Sivitz $A B$, Rosen MD, et al. A comparison of cosmetic outcomes of lacerations on the extremities and trunk using absorbable versus nonabsorbable sutures. Acad Emerg Med 2014;21:637-43.
7. Diener MK, Knebel P, Kieser M, et al. Effectiveness of triclosan-coated PDS Plus versus uncoated PDS II sutures for prevention of surgical site infection after abdominal wall closure: the randomised controlled PROUD trial. Lancet 2014;384:14252.

8. Lalani GG, Schricker AA, Salcedo J, et al. Cardiac device implant skin closure with a novel adjustable, coaptive tape-based device. Pacing Clin Electrophysiol 2016;39:1077-82.

9. Heal C, Sriharan S, Buttner PG, Kimber D. Comparing nonsterile to sterile gloves for minor surgery: a prospective randomised controlled non-inferiority trial. Med J Aust 2015;202: 27-31.

10. Brewer JD, Gonzalez AB, Baum CL, et al. Comparison of sterile vs nonsterile gloves in cutaneous surgery and common outpatient dental procedures: a systematic review and meta-analysis. JAMA Dermatol 2016;152:1008-14.

11. Perelman VS, Francis GJ, Rutledge T, Foote J, Martino F, Dranitsaris $\mathrm{G}$. Sterile versus nonsterile gloves for repair of uncomplicated lacerations in the emergency department: a randomized controlled trial. Ann Emerg Med 2004;43:362-70.

12. FLOW Investigators, Bhandari $M_{1}$ Jeray $\mathrm{KJ}$, et al. A trial of wound irrigation in the initial management of open fracture wounds. N Engl J Med 2015;373:2629-41.

13. Ghafouri HB, Zare M, Bazrafshan A, Abazarian N, Ramim T. Randomized, controlled trial of povidone-iodine to reduce simple traumatic wound infections in the emergency department. Injury 2016;47:1913-8.

14. Gurusamy KS, Toon CD, Allen VB, Davidson BR. Continuous versus interrupted skin sutures for non-obstetric surgery. Cochrane Database Syst Rev 2014;2:CD010365.

15. Min JH, Park KH, Choi HL, et al. Usefulness of direct W-plasty application to wound debridement for minimizing scar formation in the ED. Am J Emerg Med 2017;35:1804-9.

16. Deerenberg EB, Harlaar JJ, Steyerberg EW, et al. Small bites versus large bites for closure of abdominal midline incisions (STITCH): a double-blind, multicentre, randomised controlled trial. Lancet 2015;386:1254-60.

17. Webber J, Ilahi N, Lloyd G. Retrospective analysis of process times for lacerations of the face in children: procedural sedation or general anaesthesia? Br J Oral Maxillofac Surg 2016; 54:715-6.

18. Rubinstein O, Barkan S, Breitbart R, et al. Efficacy of oral ketamine compared to midazolam for sedation of children undergoing laceration repair: A double-blind, randomized, controlled trial. Medicine (Baltimore) 2016;95:e3984.

19. Neville DN, Hayes KR, Ivan Y, McDowell ER, Pitetti RD. Double-blind randomized controlled trial of intranasal dexmedeto- 
midine versus intranasal midazolam as anxiolysis prior to pediatric laceration repair in the emergency department. Acad Emerg Med 2016;23:910-7.

20. Vandamme $E$, Lemoyne $S$, van der Gucht $A$, de Cock $P$, van de Voorde P. LAT gel for laceration repair in the emergency department: not only for children? Eur J Emerg Med 2017;24: 55-9.

21. Park SW, Oh TS, Choi JW, et al. Topical EMLA cream as a pretreatment for facial lacerations. Arch Plast Surg 2015;42:2833.

22. Tayeb BO, Eidelman A, Eidelman CL, McNicol ED, Carr DB. Topical anaesthetics for pain control during repair of dermal laceration. Cochrane Database Syst Rev 2017;2:CD005364.

23. Sherman JM, Sheppard P, Hoppa E, Krief W, Avarello J. Let Us Use LET: A Quality Improvement Initiative. Pediatr Emerg Care 2016;32:440-3.

24. Mahshidfar B, Cheraghi Shevi S, Abbasi M, et al. Ice reduces needle-stick pain associated with local anesthetic injection. Anesth Pain Med 2016;6:e38293.

25. Song J, Kim H, Park E, et al. Pre-emptive ice cube cryotherapy for reducing pain from local anaesthetic injections for simple lacerations: a randomised controlled trial. Emerg Med J 2018; 35:103-7.

26. Brogan GX Jr, Giarrusso E, Hollander JE, Cassara G, Maranga MC, Thode HC. Comparison of plain, warmed, and buffered lidocaine for anesthesia of traumatic wounds. Ann Emerg Med 1995;26:121-5.

27. Masters JE. Randomised control trial of $\mathrm{pH}$ buffered lignocaine with adrenaline in outpatient operations. Br J Plast Surg 1998; 51:385-7.

28. Mader TJ, Playe SJ, Garb JL. Reducing the pain of local anesthetic infiltration: warming and buffering have a synergistic effect. Ann Emerg Med 1994;23:550-4.

29. Bartfield JM, Gennis P, Barbera J, Breuer B, Gallagher EJ. Buffered versus plain lidocaine as a local anesthetic for simple laceration repair. Ann Emerg Med 1990;19:1387-9.

30. Lundbom JS, Tangen LF, Wago KJ, et al. The influence of Lidocaine temperature on pain during subcutaneous injection. J Plast Surg Hand Surg 2017;51:118-21.

31. Wago KJ, Skarsvag TI, Lundbom JS, et al. The importance of needle gauge for pain during injection of lidocaine. J Plast Surg Hand Surg 2016;50:115-8.

32. Tangen LF, Lundbom JS, Skarsvag $\mathrm{Tl}$, et al. The influence of injection speed on pain during injection of local anaesthetic. J Plast Surg Hand Surg 2016;50:7-9.

33. Okur OM, Şener A, Kavakli HS, et al. Two injection digital block versus single subcutaneous palmar injection block for finger lacerations. Eur J Trauma Emerg Surg 2017;43:863-8.

34. Hashemian AM, Omraninava A, Kakhki AD, et al. Effectiveness of local anesthesia with lidocaine in chronic opium abusers. $J$ Emerg Trauma Shock 2014;7:301-4.

35. Roodsari GS, Zahedi F, Zehtabchi S. The risk of wound infection after simple hand laceration. World J Emerg Med 2015;6: 44-7.

36. Sirijatuphat $R$, Siritongtaworn $P$, Sripojtham $V$, Boonyasiri $A_{t}$ Thamlikitkul V. Bacterial contamination of fresh traumatic wounds at Trauma Center, Siriraj Hospital, Bangkok, Thailand. J Med Assoc Thai 2014;97 Suppl 3:S20-5.

37. Berwald N, Khan F, Zehtabchi S. Antibiotic prophylaxis for ED patients with simple hand lacerations: a feasibility randomized controlled trial. Am J Emerg Med 2014;32:768-71.

38. Tabaka ME, Quinn JV, Kohn MA, Polevoi SK. Predictors of infection from dog bite wounds: which patients may benefit from prophylactic antibiotics? Emerg Med J 2015;32:860-3.

39. Block L, Gosain A, King TW. Emerging therapies for scar prevention. Adv Wound Care (New Rochelle) 2015;4:607-14.

40. Jull AB, Cullum N, Dumville JC, Westby MJ, Deshpande S, Walker N. Honey as a topical treatment for wounds. Cochrane Database Syst Rev 2015;3:CD005083.

41. Malhotra R, Ziahosseini K, Poitelea C, Litwin A, Sagili S. Effect of manuka honey on eyelid wound healing: a randomized controlled trial. Ophthalmic Plast Reconstr Surg 2017;33:268-72.

42. Thamboo A, Mulholland G, Matthews K, Ayoub N, Anderson D. Objective and subjective scar aesthetics with topical Manuka honey post-thyroidectomy: a randomized control study. World J Otorhinolaryngol Head Neck Surg 2016;2:203-7.

43. Longaker MT, Rohrich RJ, Greenberg L, et al. A randomized controlled trial of the embrace advanced scar therapy device to reduce incisional scar formation. Plast Reconstr Surg 2014; 134:536-46.

44. Heal $C$, Buettner P, Raasch $B$, et al. Can sutures get wet? Prospective randomised controlled trial of wound management in general practice. BMJ 2006;332:1053-6.

45. Toon CD, Lusuku C, Ramamoorthy R, Davidson BR, Gurusamy KS. Early versus delayed dressing removal after primary closure of clean and clean-contaminated surgical wounds. Cochrane Database Syst Rev 2015;9:CD010259.

46. Lee SJ, Cho YD, Park SJ, Kim JY, Yoon YH, Choi SH. Satisfaction with facial laceration repair by provider specialty in the emergency department. Clin Exp Emerg Med 2015;2:179-83.

47. Amanullah $\mathrm{S}$, Linakis JG, Vivier PM, Clarke-Pearson E, Steele DW. Differences in presentation and management of pediatric facial lacerations by type of health insurance. West J Emerg Med 2015;16:527-34. 
48. Rui-Feng C, Li-Song H, Ji-Bo Z, Yi-Qing J, Yu-Jie L, Yi S. Negative pressure wound therapy for serious dog bites of extremities: a prospective randomized trial. Am J Emerg Med 2016; 34:1006-10.

49. Lee JH, Jeon MS, Lee DL, Shin HK, Seul JH. Analysis of patients with facial lacerations repaired in the emergency room of a provincial hospital. Arch Plast Surg 2015;42:34-9.

50. Tschan F, Seelandt JC, Keller S, et al. Impact of case-relevant and case-irrelevant communication within the surgical team on surgical-site infection. Br J Surg 2015;102:1718-25. 